Volume 9, No.5, September - October 2020

International Journal of Advanced Trends in Computer Science and Engineering

Available Online at http://www.warse.org/IJATCSE/static/pdf/file/ijatcse269952020.pdf

https://doi.org/10.30534/ijatcse/2020/269952020

\title{
Gesture Based Medical Visualization Interface and Image Processing Methodologies to Aid and Diagnose of Lung Cancer
}

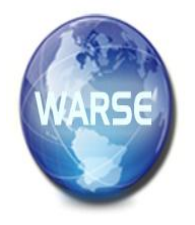

\author{
Manikanta.K.B, Deepak.D.M, Dr.C.Gopala Krishnan, Aswath Narayan.R, Sahana D S \\ Assistant Professor,GITAM School of Technology,India,11manikantareddy@ gmail.com \\ Assistant Professor, GITAM School of Technology,India,dmanjuna@ gitam.edu \\ Associate Professor,GITAM School of Technology, India,gchandra@gitam.edu \\ Professor, Brindavan College, India,aswath.narayanr@gmail.com \\ Assistant Professor,GITAM School of Technology,India,ssanthos@ gitam.edu
}

\begin{abstract}
The severity of cancer to mortality is higher around the world. So, this work aims at improving the diagnosis and planning for therapy of tumor. The main objective is to implement certain image processing methodologies in aiding the appropriate diagnosis of lung cancer and to provide sterile environment for interacting with the medical data in operation theatre during treatment. Computer Tomography (CT) gives a good resolution axial slice image of lung. But the analysis of the tumor from the raw CT data is quite impossible as the pixel characteristics of the tumor would be approximately matching its neighboring pixels. Thus a basic preprocessing algorithm is required to differentiate target from the background. Then proceeded with level set segmentation to extract tumor from the sequentially acquired multiple slices, the segmented output of each slice provide the geometric feature of the tumor. As the tumor size and shape in segmented slices are irregular, it
\end{abstract}

\section{I.INTRODUCTION}

The predominant danger on the planet is because of disease. In spite of the fact that there are numerous types of malignant growth, cellular breakdown in the lungs, i.e., the essential bronchial carcinoma is the main source of disease demise and an early discovery is urgent to the possibility for remedial treatment and to improve the endurance rate. To permit such early discovery, endeavors are in progress to build up fitting determination of tumor. The recognition of cellular breakdown in the lungs becomes testing when the objective is appended to veins or encompassed by comparable power structure like pleural divider. Accordingly, the recognition of the degree of a cellular breakdown in the lungs through division is significant for volumetric investigation. The representation of cellular breakdown in the lungs is significant for finding dependent on the its shape and its respectability to the encompassing.

The $3 \mathrm{D}$ data provided by many imaging modalities are just the rendering of whole anatomical surface, where the target to be treated will be hidden. One of the most common seems appropriate to reconstruct a three-dimensional representation of 2D images for qualitative information of tumor. The volume reconstruction using ray casting method has been included to render the volume of tumor from segmented stack of 2D slices. Then touch-less, computer aided, gesture based control of the medical images was attained using kinect sensor, which is the best tool for humancomputer interaction to maintain sterile environment. The paper elaborates about the gestures employed to interact with the medical images such as selection, drag and swipe gesture. The selection gesture used to open and close individual medical image. The drag gesture used to view the patient data along the slice image. The swipe gesture used to view variety of medical data set like 2D slices, tumor irregularity in segmented slices and 3D volume of the tumor.

Key words: Computed Tomography, Image Segmentation, Kinect, Lung Cancer, and Volume Rendering.

visualization method is direct volume rendering as the classification of object is possible through volume rendering in contrast to the surface rendering. The main parameter to be concentrated in volume reconstruction is the rendered volume should be view-dependent, such that the generated triangle mesh of the surface extracted from 2D slices are visible from all viewpoints. This paper propose a simple approach called ray casting to obtain the $3 \mathrm{D}$ reconstruction from multiple $2 \mathrm{D}$ lung carcinoma images which addresses the issue due to view dependent by occlusion culling. The second main advantage of ray casting is that it produces high quality surface from the $2 \mathrm{D}$ slices and thus increases the internal resolution and classification of objects.

An exact conclusion of tumor is fundamental on account of annulling the tumor through medical procedure. The careful arranging wants clean condition to communicate with the patient clinical record throughout medical procedure. These days, Computer data innovation is progressively entering into 
the emergency clinic area. It is significant that such innovation be utilized in a protected way to stay away from genuine mixups prompting conceivable deadly episodes. Consoles and mice are the present standard strategy for human - PC cooperation. Shockingly, it has been discovered that a typical strategy for spreading contamination starting with one individual then onto the next includes normal PC consoles and mice in concentrated consideration units utilized by specialists and medical attendants.

The distributed interactive tool aimed at surgical planning is Kinect camera. Kinect is a motion sensor device produced by Microsoft which is to create a touch-less, computer aided, gesture based control of the medical images in the operation theatre. The hand gestures captured by kinect were transformed to computational value to control the medical data. Kinect replaces the function of mice and keyboard by providing a touch less gesture based control of medical images, thus helps surgeons to locate the tumor effectively. So that neither surgeon nor technician needs to touch and interact with the computer in the operation theatre.

The remainder of the paper is organized as follows: enhancement of low contrast contour of the tumor from stack of CT bronchial carcinoma slices, localization and segmentation of tumor from all the slices, 3D volume visualization of segmented slices to know the exact shape of tumor followed by touch-less gesture based interaction with all forms of processed medical data using kinect sensor.

\section{PREVIOUS WORK}

Sunil Kumar et al [2014] expressed region growing algorithm for lung segmentation technique to separate the lung nodule from the lung lobe for better accuracy in the segmentation of lung nodule. The method was able to segment the pulmonary segments with high accuracy $(77 \%)$.

A. Prabin et al [2014] conveyed Automatic Segmentation of lung images by $\mathrm{CC}$ based region growing. The contextual clustering was proved to have better results than the manual seed point region growing method.

Leandro Cruz et al [2014] obtained information has extraordinary and corresponding qualities, consolidating calculation with visual characteristics.

Ada et al [2013] conveyed the importance of the early detection and prediction of the lung cancer survival using CT images.

Santiago Molina et al [2013] AI based strategy proposed to perceive predefined set of hand motions utilizing profundity pictures.

Chourmouzios et al [2012] expressed the choice of the parameters of the anisotropic diffusion in image processing. This explains the parameters of the anisotropic diffusion for better enhancement of images.

L. Gallo et al [2012] presents an open-source framework for a regulator free, profoundly intelligent investigation of clinical pictures.
L.M. Pedro et al [2012] present the utilization of the Kinect sensor to separate ID focuses for the investigation of human body developments.

C.Staub et al [2011] robot associates is predominantly for execution of self-governing undertakings in negligibly obtrusive medical procedure builds the activity intricacy of tele presence frameworks.

\section{III.METHODOLOGY}

The main objective of the paper is to acquire multiple gestures from human using kinect motion sensor and interact with the 2D medical images, segmented tumor slices, 3D volume as shown in Fig. 1

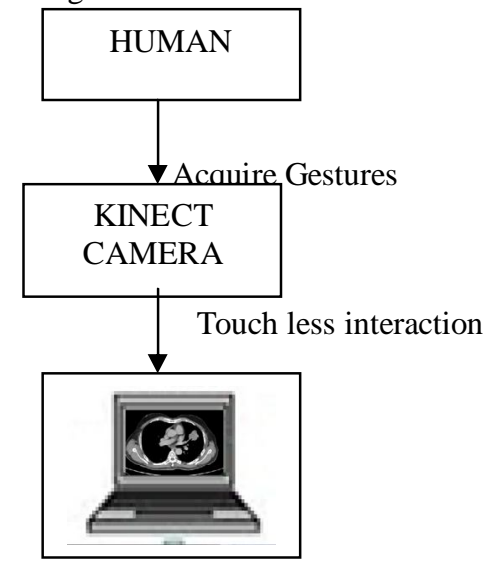

Figure.1. 3D Volume

\section{A. Overview}

Medical imaging is the technique of representing interior of the body in 2D space. The 2D image will give a good classification of anatomical structure, but the tumor which might have the same contrast like neighbour region are not distinctive in 2D images, thus certain pre processing methodologies are adopted to enhance the image followed by extracting the tumor for further analysis and visualization as shown in Figure. 2

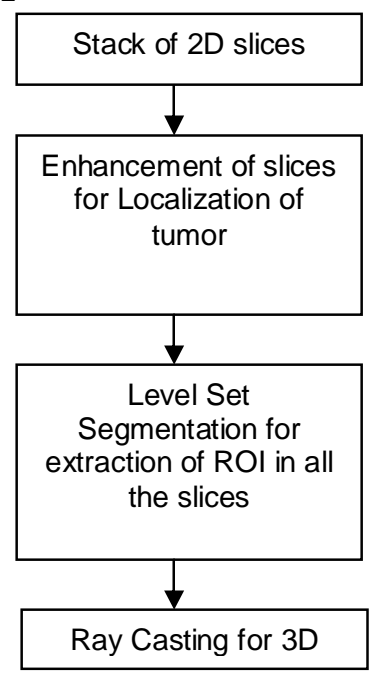

Figure.2. Visualization 


\section{B. Image Enhancement}

The aim of image enhancement is to improve the interpretability or perception of information

\section{Anisotropic diffusion}

An Anisotropic dissemination is a pre preparing procedure which accomplishes non-straight and space variation change of the info picture. The calculation holds the high recurrence edges and upgrades the low recurrence segments of the picture by applying the law of dispersion

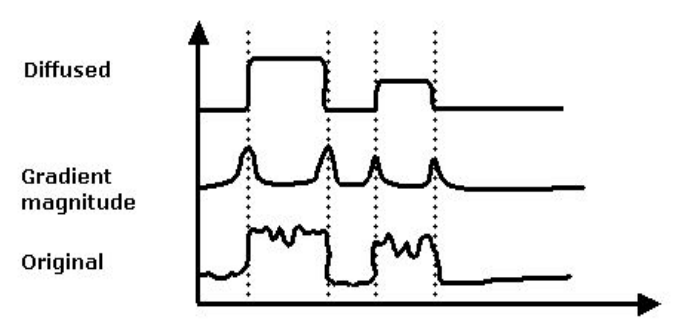

By equation,

$$
\partial \mathrm{I} / \partial \mathrm{t}=\operatorname{div}(\mathrm{c}(\mathrm{x}, \mathrm{y}, \mathrm{t}) \nabla \mathrm{I})=\nabla \mathrm{c} . \nabla \mathrm{I}+\mathrm{c}(\mathrm{x}, \mathrm{y}, \mathrm{t}) \Delta \mathrm{I}
$$

$>$ Where $\Delta$ denotes the Laplacian gradient, div is the divergence operator and $\mathrm{C}(\mathrm{x}, \mathrm{y}, \mathrm{t})$ is the diffusion coefficient.

$>\mathrm{C}(\mathrm{x}, \mathrm{y}, \mathrm{t})$ controls the rate of diffusion and is usually chosen as function of the image gradient so as to preserve edges in the image.

Algorithm for Anisotropic diffusion is as follows,

a) Read the input image

b) Assign the number of iterations

c) Set the integration constant value between $0-1 / 7$

d) Set gradient modules and conduction coefficients to preserve high contrast edges over low contrast

\section{Image Segmentation}

Segmentation of clinical pictures in 2D cut by cut has numerous helpful applications for the clinical expert, for example, perception and volume assessment of objects of intrigue, location of variations from the norm (for example tumors, polyps, and so forth.), tissue evaluation and arrangement, and more used to find articles and limits Segmentation calculations depend on one of two essential properties of power esteems: intermittence and similitude

\section{Level Set method}

The level set strategy depends on characterizing an underlying level set capacity $\mathrm{C}(\mathrm{t})$, on a two dimensional lattice $(\mathrm{x}, \mathrm{y})$. The $\mathrm{z}$ ero level capacity is moved opposite to the picture plane. As the emphasis builds the size will characterize the structure. As opposed to other division calculation, Level set strategy are utilized to locate the sharp edges with no reference layout.

The standard level set segmentation function is given by

$$
\mathrm{F}=1-\varepsilon \kappa+\beta(\nabla \varphi \cdot \nabla \nabla \mathrm{I})
$$

where ' 1 ' causes the contour to inflate inside the object. The ' $\varepsilon \kappa$ ' is the viscosity term which reduces the curvature of the contour. The final term is the edge attraction term which pulls the contour to the edges.

\section{Volume Rendering}

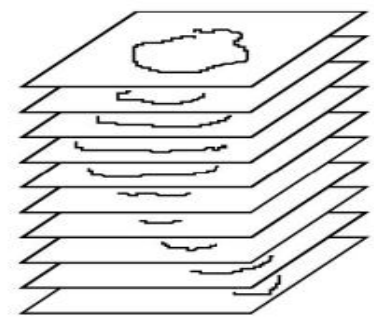

Figure.3 Reconstruction of 3D dataset by a series of 2D slices

Volume rendering of the medical images provide the quantitative information about the properties of anatomic tissues and its coordination with the surrounding. Volume is the cumulative representation of the $2 \mathrm{D}$ slices as shown in "Fig. 3 ". Volume of 2D sampled data can be rendered either by surface or volume rendering methods. Surface rendering just gives the surface representation of the volume by removing the hidden part, thus lag in the classification of internal objects. So Volume Rendering based on ray casting was used to reconstruct the 3D from slices efficiently. Volume rendering provides both surface and opacity of the volume.

Algorithm of volume rendering by ray casting,

For each pixel

1. Compute ray from eye through pixel

2. For each primitive, test for ray-object intersection

3. Shade pixel using nearest primitive

\section{E. Interaction using Kinect}

Kinect is equipped for catching profundity of every pixel in the scene by utilizing infrared producer and camera. The information gives the mathematical data about the scene. The goal of the sensor is $640 \times 480$ pixels in $30 \mathrm{~Hz}$. The skeleton picture of the client is acquired from the profundity information, which is utilized to characterize the motions. The normal motions utilized for cooperating with the clinical pictures are select, drag, swipe, pivot and zoom. The signal examination in kinect is accomplished by three significant advances like following, acknowledgment and UI. The field of view in the framework is 58 degrees level, 45 degrees vertical, 70 degrees corner to corner, and the operational range is between 0.8 meters $(2.6 \mathrm{ft})$ and 3.5 meters $(11 \mathrm{ft})$. The profundity detecting utilizes organized light technique to gauge the profundity. A known example of specks is extended from the IR laser producer. These dabs are recorded by the IR camera and afterward contrasted with the known example. Any aggravations are known to be varieties in the surface and 
can be distinguished as nearer or further away. , the profundity estimation is finished utilizing an organized light strategy. This methodology comprises in anticipating an example of pixels in the scene and catching the distortion of projection, that will permits us to register the pixel separations (profundities).

Kinect camera placed in front of a screen captures a sequence of images of the hand. The temporal information is represented by the current frame number. The two layer architecture is used. The lower level provides tracking and recognition functions, while the higher level manages the user interface.

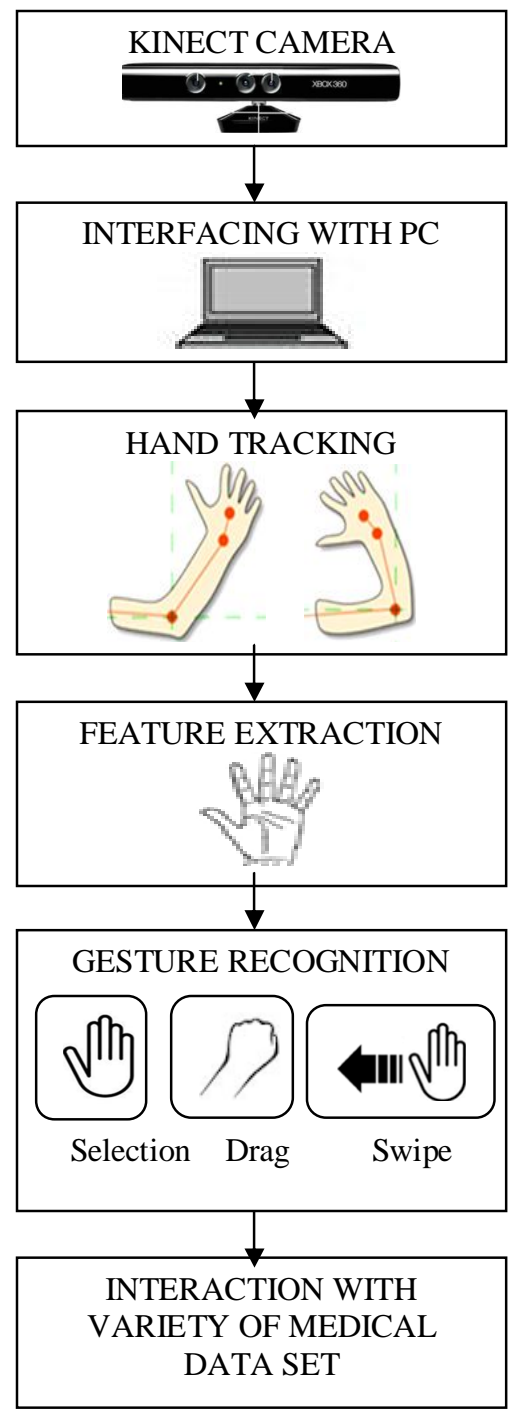

\section{Gesture Algorithm}

a) Selection gesture

- Tracking of cursor path to define the mouse movement
- Acquire the shape of hand by executing Contour and Convexity hull algorithm to track each node point of hand

- Align the joints to recognize the gesture

- Set the time delay to hold position for clicking the image.

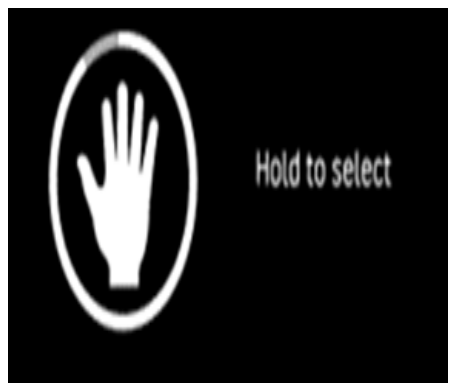

b) Swipe gesture

In the swipe gesture, the hand remains above the elbow and moves periodically from left to right.
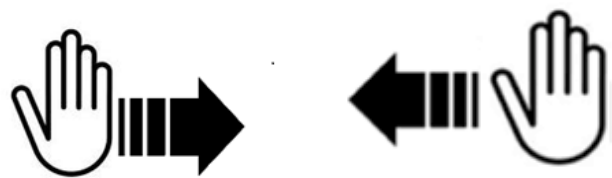

- Tracking of cursor path to define the mouse movement

- Acquire the shape of hand by executing Contour and Convexity hull algorithm to track each node point of hand

- Create a recognizer function to recognize the gesture for running the slideshow

- Assign swipe right leads to next slide and swipe left will go to previous slide

- Load the set of medical data with common path and assign index value to follow the sequence

- Create function to display previous, current and next image for sliding when swiping occurs

c) Fist gesture

The gesture is activated based on the distance measure from the centroid of the palm and the fingers. Initialize the header function to interface the Kinect with selection gesture.

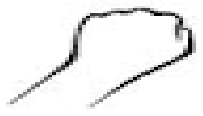

- Tracking of cursor path to define the mouse movement

- Acquire the shape of hand by executing Contour and Convexity hull algorithm to track each node point of hand

- Align the joints to recognize the gesture 
- Calculation of centroid to determine the opening and closing of fist.

- $\quad$ Set the time delay to hold position for clicking the image.

\section{IV.RESULTS AND DISCUSSION}
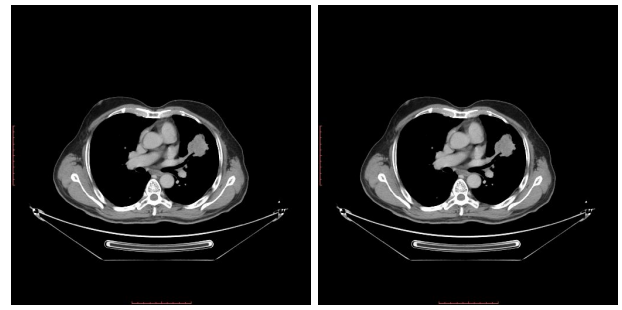

(a)

(b)

Figure.4 Image enhancement by Anisotropic Diffusion (a) Initial stage image (b) Final stage image

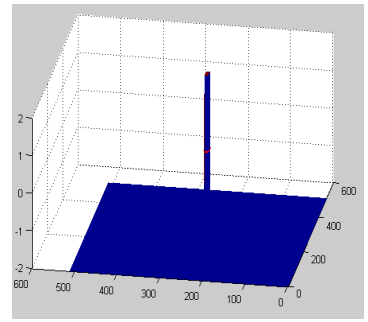

(b)
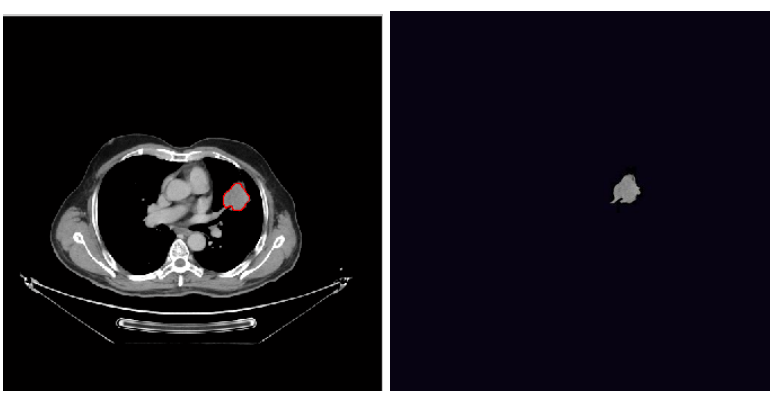

(c)

(d)

Figure.5 Level Set(a) Initial level set function (b) Final level set function after 610 iteration (c) Final zero level contour(d) segmented tumor
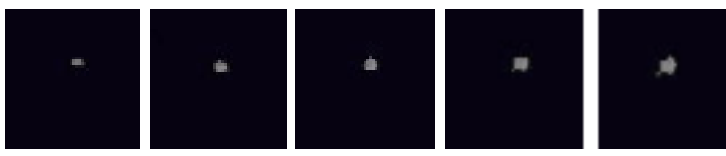

\section{3}
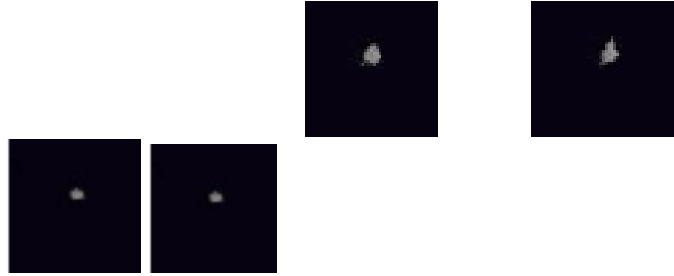

Figure.6 Segmented tumor from stack of slices

The level set segmentation was performed on all slices. Then the volume was rendered using volume rendering technique as shown in "Fig.6".

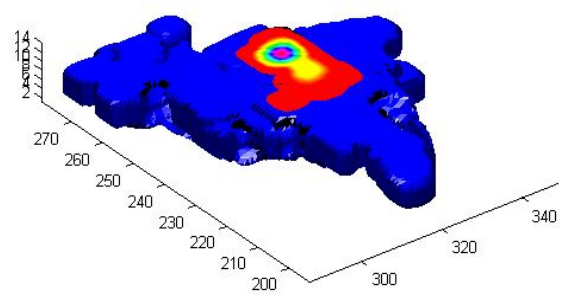

Figure.7 3D volume of bronchical carcinoma

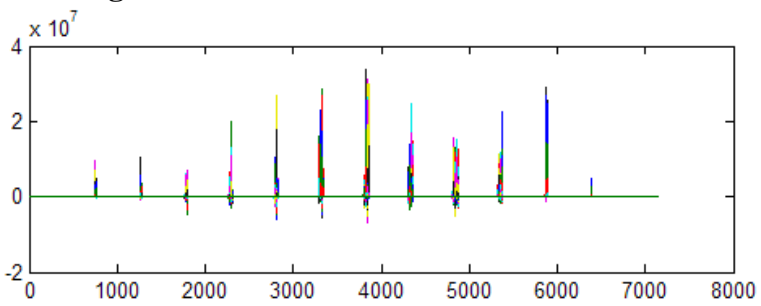

Figure.8 Performance metrics of rendered data

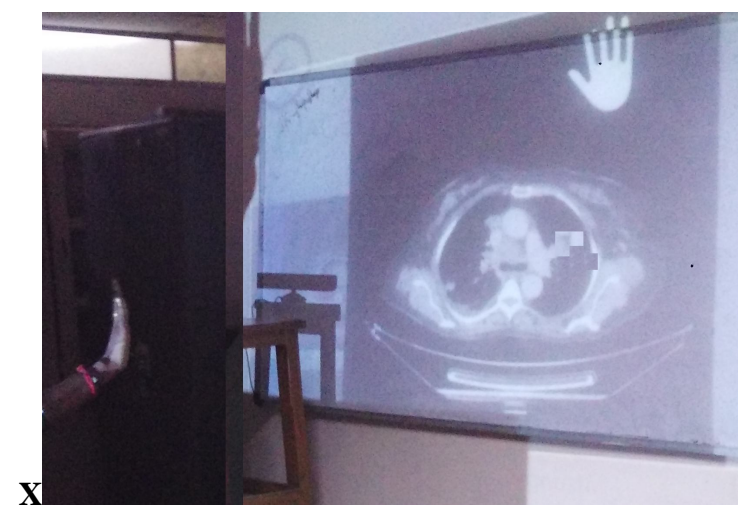

Figure.9 Gesture based control of medical image 


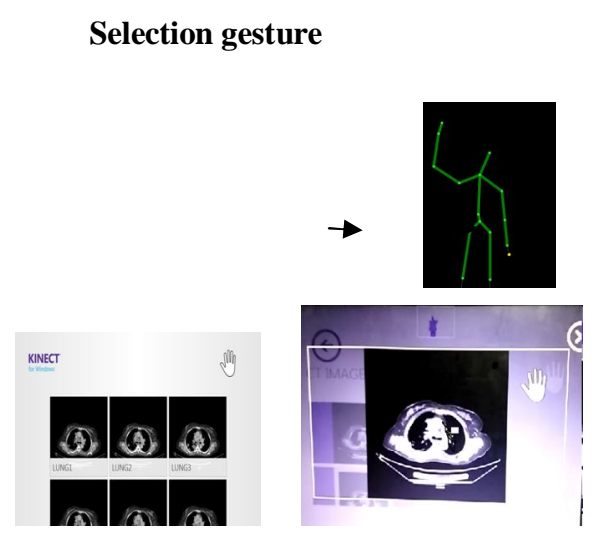

(a)

(c)

Figure.10 (a) Tracked skeletal image (b) 2D slices loaded in program (c) Opening of image using gesture

\section{Swipe Gesture}

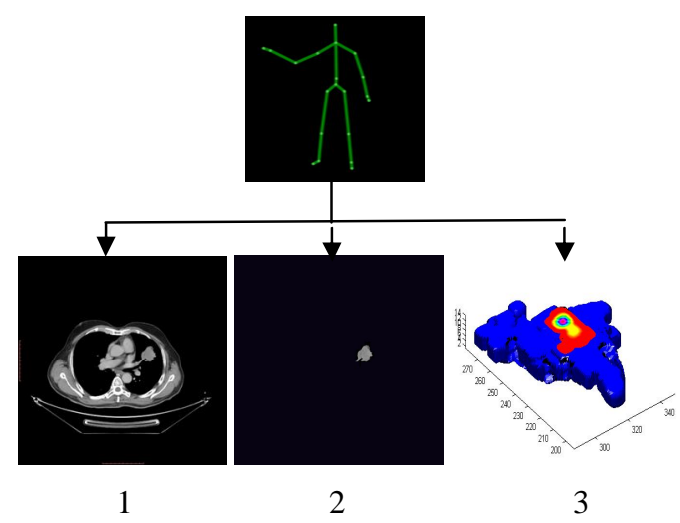

Figure.11 Track skeleton and swipe set of medical data

Drag gesture

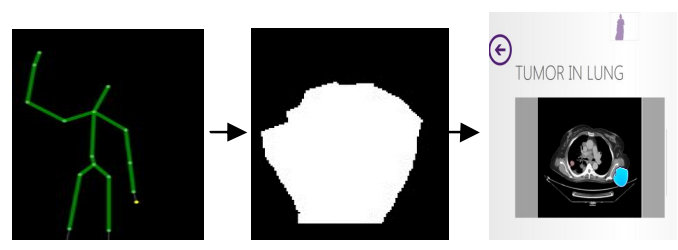

(a)

(b)

(c)

Figure.12 (a) tracked skeletal image (b) depth image of fist (c) drag image using gesture

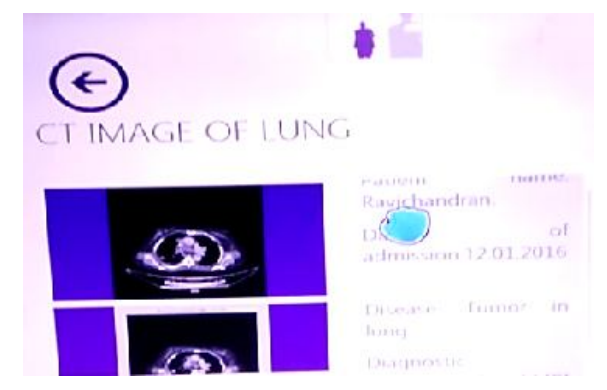

Figure.13 Drag the patient data

\section{V.CONCLUSION AND FUTURE WORK}

The diagnosis of lung tumor based on severity and depth has been achieved. The lung tumor was localised by improving the contrast and homogeneity of the input image. The direct volume rendering attains the classification of internal objects based on contrast. Futher direct volume rendering techniques like splatting and texture based rendering can be used in case of 3D space volumetice rendering. Kinect was effectively used to acquire gestures for selection, drag, swipe to interact with the medical data. Further gestures like rotate can be incorporated to control the medical data in $3 \mathrm{D}$ space.

\section{REFERENCES}

[1]. Brown, Leandro Cruz, Derek Litchi, "Kinect and RGBD images: challenges and Applications", IEEE Conference Graphics, Patterns and Images Tutorials, Nov 2013

[2]. C. Staub, Djalma Lucio, Luiz Velho, "Human - Computer Interfaces for Interaction with surgical tools in Robotic Surgery", IEEE RAS/EMBS International Conference on Biomedical Robotics and Bio-mechatronics, Dec 2013

[3]. C. R. Wren, Luigi Gallo, Chuan Lin, Xin Kong and Xinyu, "Controller- free exploration of medical image data", IEEE transaction, June 2013

[4]. Crowley, Janusz Konrad, and Prakash Ishwar, "Kinect evaluation for human body movements analysis", IEEE RAS/EMBS International Conference, July 2013

[5]. Erik Granum, Mohd Kufaisal bin Mohd Sidik,Mohd Shahrizal bin Sunar, Ismahafezi bin Ismail, "A novel gesturebased interface e for a VR simulation", Workshop on Digital Media and Digital Content Management, Sept 2012

[6]. Hervé Lahamy, Tyler Young, James C. Peyton Jone, “A study on natural interaction for human body motion using depth image data", IEEE/ASME transactions, Apr 2014.

[7]. Joshua Fabian, S. Can, B. Jensen, "Integrating the Microsoft Kinect with Simulink: real time object tracking example", IEEE/ASME transactions on mechatronics, Mar 2014.

[8]. Kathryn La Belle, Alessio Pierluigi Placitelli, Mario Ciampi, "Real time dynamic gesture recognition system based 
on depth perception for robot navigation", in IEEE Conference on Robotics and Biometrics, Dec 2013. 46

[9]. P. Pentland, "Finger tracking for the Digital Desk", proceedings of First Australasian User Interface Conference, Mar 2007.

[10]. P F., Coutaz, J., "Finger Tacking As an Input Device for Augmented Reality" International Workshop, Feb 2008.

[11]. Thomas, "Dynamic models of human motion", IEEE conference, Sept 2009.

[12] C.Gopala Krishnan, Y. Harold Robinson, Naveen Chilamkurti "Machine Learning Techniques for Speech Recognition using the Magnitude" Journal of Multimedia Information System- 7(1):33-40eISSN: 2383-7632DOI: https://doi.org/10.33851/JMIS.2020.7.1.33

[13]P.S.Apirajitha, C.Gopala Krishnan ,Dr .G.Aravind Swaminathan, Dr. E.Manohar "Enhanced Secure User Data on Cloud using Cloud Data Centre Computing and Decoy Technique "International Journal of Innovative Technology and Exploring Engineering.DOI:10.35940/ijitee.I7777.078919 (IJITEE) ISSN: 2278-3075, Volume-8 Issue-9, July 2019

[14]Gopala Krishnan C., Golden Julie E., Harold Robinson Y. (2020) Predictive Algorithm and Criteria to Perform Big Data Analytics. In: Balas V., Solanki V., Kumar R. (eds) Internet of Things and Big Data Applications. Intelligent Systems Reference Library, vol 180. Springer, Cham, https://doi.org/10.1007/978-3-030-39119-5_16

[15]Gomathi, S., Gopala Krishnan, C. Malicious Node Detection in Wireless Sensor Networks Using an Efficient Secure Data Aggregation Protocol. Wireless Pers Commun (2020). https://doi.org/10.1007/s11277-020-07291-5
[16]C. Krishnan, A.Rengarajan and R.Manikandan, "Delay Reduction by Providing Location Based Services using Hybrid Cache in Peer to Peer Networks," KSII Transactions on Internet and Information Systems, vol. 9, no. 6, pp. 20782094, 2015. DOI: 10.3837/tiis.2015.06.006.

[17]C. G. Krishnan, K. Sivakumar and E. Manohar, "An Enhanced Method to Secure and Energy Effective Data Transfer in WSN using Hierarchical and Dynamic Elliptic Curve Cryptosystem," 2018 International Conference on Smart Systems and Inventive Technology (ICSSIT), Tirunelveli, India, 2018, pp. 1-7, doi: 10.1109/ICSSIT.2018.8748785.

[18]O. Pandithurai, M. Poongodi, S. P. Kumar and C. G. Krishnan, "A method to support multi-tenant as a service," 2011 Third International Conference on Advanced Computing, Chennai, 2011, pp. 157-162, doi: 10.1109/ICoAC.2011.6165166.

[19]Velu S.G., Gopala Krishnan C., Sivakumar K., Jevin J.A. (2020) Proof of Shared Ownerships and Construct A Collaborative Cloud Application. In: Balaji S., Rocha Á., Chung YN. (eds) Intelligent Communication Technologies and Virtual Mobile Networks. ICICV 2019. Lecture Notes on Data Engineering and Communications Technologies, vol 33. Springer, Cham.https://doi.org/10.1007/978-3-030-283643_51 